\title{
La web social y su impacto en las bibliotecas universitarias mexicanas
}

The social web and its impact on Mexican university libraries

Veronica Soria Ramírez (1), Violeta Soria Ramírez (1) y Apolinar Sánchez Hernández (2)

(1) Departamento de Consulta. Dirección General de Bibliotecas de la UNAM, Edificio de "Biblioteca Central" Circuito Interior s/n, Ciudad Universitaria, Deleg. Coyoacán C.P. 04510, México, D.F vsoriar@hotmail.com, enlinea@dgb.unam.mx (2) Departamento de Catálogo Colectivo de Publicaciones Periódicas. UNAM-DGB, Edificio de "Biblioteca Central", Circuito Interior s/n, Ciudad Universitaria, Deleg. Coyoacán C.P. 04510, México, D.F. asanchez@dgb.unam.mx, sanherap@gmail.com

\section{Resumen}

Análisis del impacto de la web social en las bibliotecas mexicanas ante la Web Social a partir de una revisión de los portales de las bibliotecas de algunas de las universidades públicas y privadas más representativas.

Palabras clave: Web Social. Bibliotecas universitarias. México.

\section{Introducción}

En los medios impresos y en Internet se habla cada vez más de Web Social, Web 2.0, aprendizaje colaborativo, open access, software libre, OPAC social, mash-up, recursos colaborativos, redes sociales, folksonomías, etiquetado colaborativo, blogs, wikis, beta perpetua, sindicación de contenidos, entre muchos otros términos que ya forman parte del vocabulario común de cualquier profesional de la información, además de ocupar grandes espacios en los medios de comunicación masiva y en la literatura especializada de las diversas áreas del conocimiento; la lista crece día a día y los mash-ups nativos de este entorno se multiplican.

Esta enorme actividad, esta marea de nuevos anglicismos está cambiando el mundo de las telecomunicaciones, de los medios de comunicación, del software, del entretenimiento, etc. Todo este nuevo conjunto de medios conforma lo se ha dado en llamar Web 2.0, la nueva etapa en la evolución de Internet.

Ante esta marea de vocablos que se traducen en servicios, recursos, herramientas, los profesionales de la información deben mantenerse actualizados para poder acercar a su comunidad a este cambiante escenario, en el que las aportaciones se dinamizan y las colaboraciones en red se dan a cada instante. Por otra parte, la representación de la biblioteca como institución

\begin{abstract}
The impact of the social web on Mexican university libraries is analyzed. A review is done of the web sites of the most representative public and private universities.
\end{abstract}

Keywords: Social Web. University libraries. Mexico.

en la Red es crucial para mantener y aumentar su presencia en la comunidad. Es por ello, que la utilización de las herramientas 2.0 es un medio ideal para prestar servicios, recursos, colecciones y reposicionar la imagen de la biblioteca, además de ser un excelente canal de comunicación con los usuarios.

Para la elaboración del presente documento se utilizó el Ranking Web of World Universities, primer semestre de 2009. Se presenta una revisión de los portales de bibliotecas y sistemas bibliotecarios de las 21 instituciones de educación superior de México, que de acuerdo a este ranking tienen presencia en la Red.

\section{Web 2.0}

Hoy en día se habla acerca de una Web participativa, es decir, una red en la que los usuarios interactúan más activamente. La OCDE define a la Web participativa de la siguiente forma (OCDE, 2007):

El concepto de Web participativa está basado en un Internet cada vez más influenciado por servicios Web inteligentes que permiten a los usuarios contribuir a desarrollar, calificar, colaborar y distribuir contenido de Internet y personalizar las aplicaciones.

Este término tiene una estrecha relación con el de contenidos creados (UCC por sus siglas en inglés). No existe una definición aceptada sobre 
este término, se utiliza para referirse al contenido que se encuentra disponible públicamente en Internet, que refleja algún tipo de esfuerzo creativo y que es generado fuera de las prácticas y rutinas consideradas como tradicionales (OCDE, 2007), el más obvio de sus efectos es la inmediata propagación de los contenidos a millones de personas.

La Web 2.0 también Ilamada Web Social es el resultado de una evolución que ha tenido la red en los últimos años; significa una evolución de la Web, es un cambio desde el cerebro de cada uno de los usuarios de Internet, representa una nueva configuración en la disposición de recursos, interacción entre usuarios y la conformación de redes sociales, ya que actualmente ofrece la posibilidad de utilizar una gran cantidad de recursos de software gratuito, disponible desde la propia Web para publicar información en conjunto.

En este estadio el usuario ha adquirido gran protagonismo gracias a herramientas que propician la participación y el intercambio de ideas. Pensar en red, tener acceso a grandes volúmenes de información y la diversidad de medios a los que podemos acceder resulta tan fascinante como abrumadora. En opinión de Rheingold intercambiar el know-how con gente de seis continentes en tiempo real transforma fundamentalmente la forma de compartir conocimiento al bajar dramáticamente el costo de la transacción de hacer corresponder preguntas y respuestas, el intercambio de conocimiento eficiente puede generar poder real en las comunidades científicas y empresariales, en forma tan simple como tener una conversación en línea, y más aún, los efectos se multiplican cuando este intercambio se lleva a cabo a través de grupos (Rheingold, 2002).

La Web Social sigue una filosofía con principios de lectoescritura de carácter participativo donde cada usuario puede intervenir directamente en la elección, selección y contribución de sus aportes de información dentro de cada sitio web. Además, es cooperativa, pues de esta forma se aportan ideas, preferencias, información y conocimiento; es interactiva, en la medida que a través de recursos multimedia es posible un diálogo simultáneo con usuarios; es democrática, porque bajo esta filosofía existe libertad de expresión y pensamiento, y sobre todo, libertad de flujos de información; es socializadora, porque a través de todas sus características es posible un continuo intercambio de ideas, visiones, culturas.

Estamos entonces ante un sistema abierto, donde el todo es más que la suma de sus par- tes. Así, cada usuario aporta experiencias y conocimiento a este gran cúmulo de información almacenado en millones de computadoras alrededor del mundo y el resultado sobrepasa los miles de terabytes de información almacenada en la red, pero el conocimiento que de allí podemos obtener no puede medirse.

El sistema de la Web 2.0 consiste en escoger un diálogo intercultural que asume la alteridad y la diferencia creando comunidades más allá de los límites geográficos que puedan existir (Villouta, 2008).

Las características clave que definen un servicio Web 2.0 pueden resumirse del siguiente modo:

- Concepto de servicio, no tanto de software empaquetado, con escalabilidad rentable.

- Control sobre fuentes de datos únicos que se enriquecen a medida que más gente las utiliza.

- Confianza en los usuarios como codesarrolladores. El usuario es innovador, es un prosumidor de contenidos, acrónimo que procede de la fusión de dos palabras producer (productor) y consumer (consumidor). El concepto de prosumidor fue anticipado por Marshall McLuhan y Barrington Nevitt, quienes en el libro Take Today (1972) afirmaron que la tecnología electrónica permitiría asumir simultáneamente los roles de productor y distribuidor de contenidos (Islas-Carmona, 2008).

- Aprovechamiento de la inteligencia colectiva, orientación social y colaboradora.

- Procesos descentralizados y distribuidos.

- Etiquetado colectivo y colaborador de información (folkosomías).

- Explotación de "the long tail" (la larga cola) mediante el autoservicio del cliente.

- Software no limitado a un solo dispositivo, interfaces de usuario, modelos de desarrollo y modelos de negocio ligeros

Un servicio Web 2.0 se integra por módulos o fragmentos de aplicaciones que son acoplados entre sí de manera independiente. Gracias a esta funcionalidad es factible construir nuevas aplicaciones aprovechando las bondades de servicios provenientes de diferentes fuentes (mash-up, mezcla).

Es así como la Web se presenta como un modelo de consumo y de creación abierta para todo, donde se van realizando mejoras sustanciales presentes en servicios cada vez más amigables para el usuario. Esta modalidad dota a los servicios Web 2.0 de flexibilidad y reduce 
los riesgos de conflicto en caso de cambios en uno de los módulos. Por otro lado, los programas son concebidos cada vez más como servicios que como productos. En vez de ser modificados en profundidad periódica y constantemente (como las versiones del software comercial), estos se suelen ajustar y actualizar de manera casi constante, surgiendo de esta forma la beta perpetua.

La tecnología modificó la cultura de las personas y los desarrollos pasaron del copyright al copyleft otorgando al usuario el poder para ejercer las cuatro libertades sobre el software (ejecutar, estudiar, modificar y distribuir). Así, el antiguo sistema de control ahora tiene compañía, coexiste -o está aprendiendo a convivircon una filosofía radicalmente opuesta: Se trata de un sistema que garantiza compartir libremente la información y no busca sancionar a todo aquel que pudiera transgredir la autoridad del creador del software que anteriormente figuraba como "dueño del conocimiento". Esta libertad para compartir sobrepasó la barrera del software para regir también a los contenidos, donde iniciativas como Creative Commons, tienen espacio en la Fundación Copyleft, situación lógica ya que, si no publicamos, nuestra producción intelectual es como si no existiera. Éstas iniciativas evidencian el cambio en la manera de concebir la Web.

El éxito de la Web 2.0 se debe en gran parte al uso de tecnologías ligeras como AJAX (Asynchronous JavaScript and $\mathrm{XML}$ ), que permiten desarrollar interfaces Web ligeras para estos nuevos servicios optimizando las transacciones entre las aplicaciones residentes en el navegador y el servidor. El protocolo RSS está siendo también esencial para el desarrollo de esta filosofía de construcción de servicios. Las RSS se conocen popularmente porque facilitan la sindicación del contenido de blogs, pero en la actualidad se utilizan también para sindicar podcast, servicios de vídeo y noticias, así como para agregar servicios en sí; de hecho, se le ha comenzado a conocer como el "pipe" de Internet o el "aglutinante" para crear servicios.

Existe una inmensurable cantidad de productosrecursos-servicios y aplicaciones Web 2.0. La lista se incrementa día a día. Su incorporación, e integración en el comportamiento social está permitiendo al usuario no solo conectarse a la red, sino participar en la construcción de la inteligencia global: "Nos encontramos en plena tercera fase, la condición inalámbrica, donde todo el sistema electrónico sensorial, muscular y cognitivo regresa al cuerpo del usuario" (Kerchiove, 2005), donde el usuario accede a un proceso dinámico y colectivo que le permite reconocer en la virtualidad la posibilidad de compartir y aprender. A raíz de ello, tenemos servicios orientados a la participación, no al uso, donde los usuarios aportan un valor esencial al servicio, y la información es compartida.

En las siguientes líneas sólo dedicaremos algunos párrafos a señalar las que tienen mayor popularidad entre los cibernautas.

\section{Servicios 2.0}

El devenir histórico y el acelerado desarrollo tecnológico convergen hoy en día. Las TIC han permitido entrever el conjunto indisoluble que representan tecnología, comunicación y sociedad, y que permite de manera innovadora el trabajo digital colaborativo a través de las comunidades virtuales, donde la cultura hace referencia a la propia cultura y la organización social se basa en el flujo de información. Como mencionan Arroyo y Merlo (2006) las características de la Web 2.0 se basan en una arquitectura de participación. De esta forma, las sociedades se organizan en torno a redes, modificando la operación y los resultados de los procesos de producción, la experiencia, el poder y la cultura (Castells, 1999).

Los llamados marcadores sociales o aplicaciones de social bookmarking permiten gestionar nuestros favoritos (sitios Web, recursos o contenidos que nos han parecido de interés) desde cualquier computadora conectada a Internet. De ese modo, vamos clasificando de forma colaborativa con etiquetas conceptuales los recursos de la Web, creando entre todos los usuarios taxonomías de etiquetas y materiales sin jerarquías (folksonomías, "clasificación gestionada por el pueblo" o de forma democrática).

Las redes siempre han existido como frutoconsecuencia de las relaciones humanas. Surgieron de la convivencia diaria entre las personas: convivir implica vivir con, vivir con los otros, y ese vivir con los otros implica mucho más que un conjunto de individuos compartiendo una misma área geográfica.

En términos generales, el concepto de red se utiliza para hacer referencia a dos fenómenos: por un lado, se consideran redes todos los conjuntos de interacciones que se dan de forma espontánea; $y$, por el otro, las redes pretenden organizar esas interacciones espontáneas con un cierto grado de formalidad, en el sentido de establecer intereses, problemáticas, preguntas y fines comunes.

El concepto de red, en su forma más básica y derivado de la antropología, hace referencia a un campo social constituido por relaciones entre 
personas, con una corriente permanente de intercambio recíproco. Se define como el conjunto de relaciones (los grafos sociales) donde los nodos (actores o participantes) interactúan en torno a un interés común. El reunirse en torno al interés común (el objeto social) es lo que hace una comunidad. Al ser llevado al plano macrosocial conserva la esencia de este concepto y se redefine como organización social que coordina actores autónomos, quienes voluntariamente intercambian información, bienes o servicios con el fin de lograr un resultado conjunto (Messner, 1999).

En 2002 comienzan a aparecer sitios Web promocionando las redes de "círculos de amigos" en línea, cuando el término se empleaba para describir las relaciones en las comunidades virtuales, y se hizo popular en 2003 con la llegada de sitios como "Friendstar", MySpace. La popularidad de estos sitios creció rápidamente e íconos de Internet han lanzado sus propios servicios; Google lanzó Orkut el 22 de enero de 2004, Yahoo creó su espacio de red social en 2005.

Hoy en día, cuando se menciona este término lo extrapolamos a Internet automáticamente. Nuestro referente inmediato es la Web 2.0, por antonomasia Facebook, MySpace, Top of Mind, Flickr, etc., por sólo mencionar algunas.

La estructura de la red se caracteriza principalmente por ser flexible, horizontal e informal, lo que permite que se expanda integrando nuevos actores o nodos "miembros" mientras comparten los mismos códigos de comunicación, valores, objetivos o metas de actuación (Castells, 1999). Por otra parte, fomentan las relaciones entre sistemas diferenciados sin que sean regidos por un centro o desde arriba — sin jerarquías-. Además, facilitan su disolución o transformación en la medida en que se cumplen los objetivos colectivos y retoman la importancia de las relaciones humanas para crear lazos de solidaridad, confianza y reciprocidad.

En líneas generales se pueden identificar tres grandes tipos de redes, aunque el límite que diferencia a unas de otras es a veces difuso. En este sentido podemos hablar de:

- redes de propósito general o de masas o megacomunidades (por ejemplo, Facebook, MySpace, Twitter);

- redes abiertas para compartir archivos, sean en formato videoclip, presentación, fotografías... (por ejemplo, YouTube, SlideShare, Snips, Flirck, ...);
- redes temáticas o microcomunidades con un interés específico (por ejemplo, Ning, Elgg, GROU.PS, Google Groups).

Las bitácoras, weblogs o blogs, son sitios web estructurados originalmente como diarios personales, basados en enlaces, noticias y opiniones que se actualizan de modo regular, escritos con un estilo informal y subjetivo. La blogósfera constituye uno de los espacios más dinámicos de la Red. Las bitácoras han dejado de ser exclusivamente diarios personales, se han convertido en nuevos medios de comunicación. Los blogs se han consolidado como una herramientas de comunicación social interactiva. Son un elemento indispensable en los planteamientos de la Biblioteca 2.0, pero también ofrecen ya hoy un medio de comunicación profesional.

El hombre es siempre un narrador de historias; vive rodeado de sus historias y de las ajenas, ve a través de ellas todo lo que sucede y trata de vivir su vida como si la contara. (Jean Paul Sartre, La náusea)

\section{Web 2.0 y bibliotecas}

Los cambios en materia de tecnologías de información y comunicación, producidos a finales del siglo pasado y principios de este, han sido aprovechados satisfactoriamente por las bibliotecas para potenciar su imagen, recursos, productos y servicios, estableciendo así una relación comunicativa más inmediata y expedita con su comunidad de usuarios.

La biblioteca adoptó la tecnología porque le simplificaba una serie de rutinarias operaciones manuales; además, su uso estaba legitimado por instituciones bibliotecarias relevantes. Hoy, en cambio, la Web Social le propone a la biblioteca la interrelación con su comunidad de usuarios, que la motive y oriente no solo en la búsqueda y captura de información y conocimiento, sino en la gestión, concreción, expresión y transmisión de sus propias inquietudes y saberes. Tal vez ha faltado en beneficio de este propósito el aval de instituciones legitimantes. No obstante, una profunda mirada al interior de nuestras unidades, un tiempo de reflexión acerca de los objetivos, misión y visión de la biblioteca y los bibliotecarios, podrían despejarnos algunas dudas y mostrarnos, sin temor, nuevos horizontes.

Lee King (2007) en Library 2.0 ripples another go at the graph hace un recorrido por el paso de las bibliotecas tradicional a una biblioteca 2.0. Su metáfora evolutiva sigue el ritmo de las ondas en el agua, son seis estadios en los que divide ese oleaje: 
1. Biblioteca tradicional: todo empieza aquí.

2. Mejorando la biblioteca tradicional: Las bibliotecas se dan cuenta de que los motores de búsqueda, las bases de datos online y la referencia a través del correo electrónico pueden mejorar sus servicios tradicionales, sin embargo, aun no perciben que estos son solo herramientas que por sí solas no mejoran los servicios tradicionales.

3. Cambio y escrutinio del horizonte: Las bibliotecas se dan cuenta de que en el siglo XXI los servicios permanecen por sí mismos. Empiezan a leer y ver nuevas tendencias, y se dan cuenta que para responder a las demandas actuales y futuras tienen que cambiar. Están listas para experimentar con herramientas emergentes.

4. Proyectos pilotos: La biblioteca experimenta con estas herramientas, aprende sobre la biblioteca 2.0, comienza a editar blogs; al personal se le permite jugar con estas herramientas, conservar sus conversaciones digitales, experimentar con videos, podcasting, etc.

5. Participación del usuario: la biblioteca comienza a desarrollar su sede Web como una sucursal digital que ofrece a los usuarios participación en vez de solo información. Las conversaciones tienen lugar en esta contraparte digital de muy diferentes formas. La biblioteca forma a su personal para que pueda interactuar mediante en este entorno digital, percatándose de que este cambio radical es una mejora. Los nuevos lugares Web se convierten en una conversación con adiciones más que con ediciones.

6. Comunidad involucrada: Este es el objetivo, la biblioteca y su comunidad local crean una comunidad digital a través de su sucursal digital. La biblioteca anima a la comunidad a participar en tiempo real y la sucursal digital se reconoce como la sucursal real.

En palabras de Habid (2006) la Biblioteca 2.0 no es "solo" tecnología, pero "también" es tecnología. Dicha tecnología es muy sencilla en su desarrollo y utilización, permite sin problemas colaterales seleccionar y distribuir información. Hay miles de servicios que escudriñan cada nuevo pedazo de información en línea, utilizando esta información, premezclándola con nuevas maneras y pasándola a otros servicios. Las organizaciones están utilizando la información provista por incontable número de personas que navegan por la red y que utilizan sus servicios y constituidas sobre herramientas creadas por otra gente (Zitt, 2007).
Las bibliotecas deben observar seria, serena y sabiamente su entorno, el real y el virtual. Tanto en uno como en otro se producen cambios continuamente. Ahí están los recursos de la Web 2.0: bancos de imágenes, de sonidos, de textos producidos por nuestros propios usuarios. Es momento de celebrar el hecho comunicativo y de compartir y gestionar información y conocimientos. Es necesario reconocer que los usuarios necesitan conversar entre ellos sobre los libros que leen, las películas que ven, los artículos que citan, etc. (Margaix-Arnal, 2007): "si las bibliotecas no les ofrecen un espacio para conversar, lo buscarán en otro sitio y lo encontrarán a un clic de distancia".

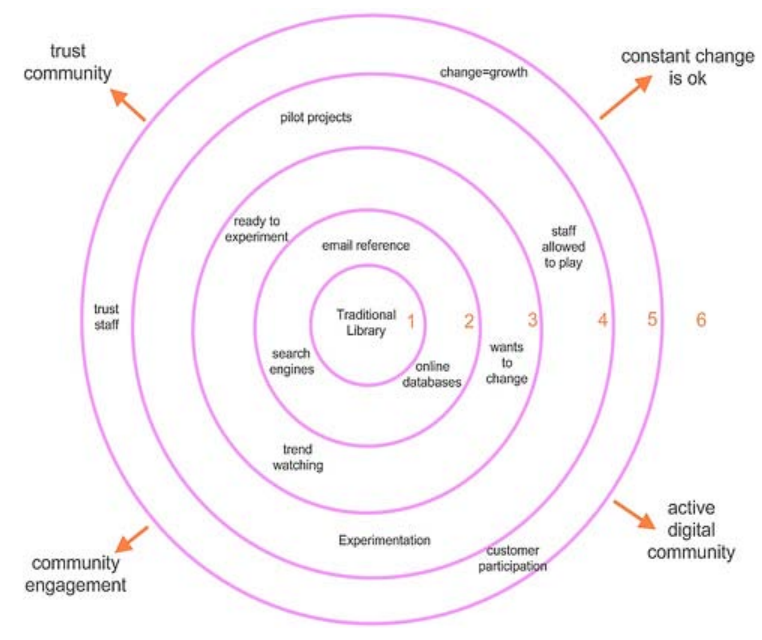

Figura 1. David Lee King (2997). Library 2.0 ripples: another go at the graph

El éxito de los sitios de redes sociales en los últimos años ha llevado a muchas bibliotecas a querer estar presentes también en ellas de diferentes formas. Parece ser que el gran impulso vino con la gran popularidad de MySpace entre los adolescentes estadounidenses, lo que hizo pensar a los profesionales ¿por qué no acercarse a este público a través de sus mismos espacios? Numerosas bibliotecas públicas de ese país crearon sus perfiles en esta red social, algunas bibliotecas han decidido mantener incluso varios perfiles en diferentes servicios. Esta última red es preferida especialmente por las universidades, dado su origen universitario, y no sólo en Estados Unidos, sino también en Europa. La existencia de perfiles bibliotecarios conlleva en algunos casos la creación de grupos temáticos de carácter profesional en el seno de estas redes generales.

Las posibilidades de las aplicaciones de Facebook también han sido explotadas por varias bibliotecas, especialmente para ofrecer acceso 
directo a sus catálogos sin tener que salir de la plataforma de Facebook, facilitando así el acceso. O ejemplos de grandes catálogos como Worldcat COPAC (que incluye los catálogos de las mayores bibliotecas universitarias y nacionales de Reino Unido e Irlanda), etc.

También se han empleado este tipo de aplicaciones para crear servicios de referencia en línea desde los que se responde a las preguntas de los usuarios. Es el caso de Ask librarian, una aplicación de los bibliotecarios de Florida que se puede personalizar con el logo de cada biblioteca.

Si centramos la mirada en el intercambio profesional con otros colegas, encontraremos a numerosos profesionales que han creado sus perfiles y participan activamente en grupos de debate o simplemente intercambiando experiencias con otros colegas. Otros han preferido crear su propia red social en servicios gratuitos como Ning o la comunidad Información 2.0

Este concepto arropa nuevas estrategias de planificación bibliotecaria en las que se vuelve sumamente relevante la participación directa de sus usuarios. Las bibliotecas están en un proceso de cambio sin lugar a dudas; deben estrechar lazos con cada individuo de su comunidad y sostenerse como una alternativa confiable de información, capaz de cubrir cada una de sus necesidades. Los cambios tecnológicos afectan a toda la estructura de las unidades de información, de ahí que su personal deba también prepararse para atender satisfactoriamente a los usuarios, ofreciéndoles nuestros tradicionales y nuevos servicios, usando para ello todos los recursos emergentes y funcionales. Se debe inculcar en la cultura bibliotecaria, tanto en la gerencia como en los niveles operativos, que la tecnología no puede nunca reemplazar un servicio pero puede mejorarlo.

\section{Web 2.0 en bibliotecas universitarias mexicanas}

Para abordar este punto, se recurrió a efectuar una revisión de los portales de las bibliotecas de algunas de las universidades públicas y privadas en México (Tabla 1). Dicha tabla es obtenida del Ranking Mundial de Universidades en la Web WEBOMETRICS (1) que se publica semestralmente (enero-junio) desde el año 2004. n ella se analizan más de 16,000 Instituciones de Educación Superior de todo el mundo. La presencia Web mide la actividad y visibilidad de las instituciones y es un buen indicador del impacto y prestigio de las universidades.

\begin{tabular}{|c|c|c|}
\hline Posición & Universidad & Ranking Mundial \\
\hline 1 & $\begin{array}{l}\text { Universidad Nacional Autónoma } \\
\text { de México }\end{array}$ & 44 \\
\hline 2 & Tecnológico de Monterrey & 406 \\
\hline 3 & Universidad de Guadalajara & 588 \\
\hline 4 & $\begin{array}{l}\text { Universidad Autónoma } \\
\text { Metropolitana }\end{array}$ & 652 \\
\hline 5 & $\begin{array}{l}\text { Universidad Autónoma del } \\
\text { Estado de México }\end{array}$ & 843 \\
\hline 6 & Instituto Politécnico Nacional & 943 \\
\hline 7 & Universidad de Sonora & 1260 \\
\hline 8 & Universidad de Colima & 1285 \\
\hline 9 & $\begin{array}{l}\text { Universidad Autónoma de Baja } \\
\text { California }\end{array}$ & 1322 \\
\hline 10 & Universidad de las Américas & 1325 \\
\hline 11 & Universidad Iberoamericana & 1352 \\
\hline 12 & $\begin{array}{l}\text { Instituto Tecnológico Autónomo } \\
\text { de México }\end{array}$ & 1358 \\
\hline 13 & $\begin{array}{l}\text { Universidad Autónoma de } \\
\text { Tamaulipas }\end{array}$ & 1387 \\
\hline 14 & Universidad Veracruzana & 1458 \\
\hline 15 & $\begin{array}{l}\text { Universidad Autónoma de } \\
\text { Puebla }\end{array}$ & 1463 \\
\hline 16 & $\begin{array}{l}\text { Centro de Investigación y } \\
\text { _Docencia Económicas }\end{array}$ & 1512 \\
\hline 17 & El Colegio de Mëxico & 1533 \\
\hline 18 & $\begin{array}{l}\text { Universidad Michoacana de San } \\
\text { Nicolás de Hidalgo }\end{array}$ & 1574 \\
\hline 19 & $\begin{array}{l}\text { Universidad Autónoma de } \\
\text { Ciudad Juárez }\end{array}$ & 1720 \\
\hline 20 & $\begin{array}{l}\text { Universidad Autónoma de } \\
\text { Chihuahua }\end{array}$ & 1794 \\
\hline 21 & $\begin{array}{l}\text { Instituto Tecnológico y de } \\
\text { Estudios Superiores de } \\
\text { Occidente }\end{array}$ & 1824 \\
\hline
\end{tabular}

Tabla I. Instituciones mexicanas incluidas en el Webometrics

La tabla en cuestión arrojó 21 instituciones mexicanas de enseñanza superior localizadas en la Webometrics, mismas que fueron exploradas.

El objetivo de la revisión era identificar en su portal aquellos servicios, herramientas o aplicaciones 2.0 que utilizan las bibliotecas para comunicarse con sus usuarios y identificar sí ellos son usuarios de tales servicios.

Dentro del análisis cuantitativo realizado en Internet, el $67 \%$ de las instituciones mexicanas son públicas, en tanto que el restante $33 \%$ pertenecen a la iniciativa privada. En lo referente a que éstas ofrezcan servicios de Biblioteca Digital, se tiene que el $85 \%$ lo brindan, en tanto que el $15 \%$ restante aún no lo ofrece. De igual manera, el $25 \%$ cuentan con RSS y el $75 \%$ restante no lo incluye. Otro dato que se obtuvo es que el $25 \%$ dispone de algún tipo de red, y el restante $75 \%$ aún no lo indica. 


\section{Consideraciones finales}

Como acertadamente señala (Castells, 2003) "la historia de la tecnología demuestra claramente que la contribución de los usuarios es crucial en la producción, ya que la adaptan a sus propios usos y valores y, en último término, transforman la propia tecnología". La Web 2.0 ha transformado a los pasivos consumidores en activos prosumidores, que han impulsado importantes transformaciones en el mercado, convirtiendo a Internet en un medio ideal para conversar, como el idóneo multiplicador del capital intelectual.

Los bibliotecarios como profesionales de la información comprometidos con nuestra comunidad no podemos ni debemos quedar al margen de dicho fenómeno. En el caso que nos ocupa, las bibliotecas universitarias mexicanas aun están distantes de incorporar estos recursos y servicios a sus comunidades. Si bien es cierto que no se puede descartar que la institución como tal aun no integre estas modalidades a su sitio de Internet, muchos de los profesionales de estas instituciones se encuentran inmersos en estos escenarios y, desde su tarea diaria, informan y forman al usuario con estos nuevos signos de los tiempos, a la par que se mantienen actualizados en estas temáticas y aportan sus experiencia particulares, socializando con sus colegas.

Es la integración entre tecnología, usuario y servicio lo que permitirá, con el transcurso del tiempo, optimizar los servicios a la medida establecida por sus usuarios. Siendo así, ¿por que no comenzar a trabajar desde ya en este sentido? Nuestras bibliotecas deben sumarse, adaptar, desarrollar y experimentar más que asumir posiciones conservadoras; con la utilización de herramientas y servicios que impulsan y fortalecen la interactividad y la participación, se puede construir un salto tecnológico que permita insertar un nuevo diseño, una capacidad comunicativa con los usuarios mucho mas potente y una pujanza creativa considerable con interacciones y con aplicación en todo el quehacer bibliotecario.

Fundamental es considerar que la biblioteca 2.0 no sólo se desarrolla en un entorno virtual, sino principalmente en uno real. 2.0 es principalmente una actitud, y, si bien es cierto que la mayoría de los servicios se ofrecen a través de la Web, es imprescindible contemplar que el desarrollo de estos dependerá esencialmente de las capacidades de los profesionales y personal de servicio de las bibliotecas; es decir, de cómo estos asuman el desafío de cómo usar las nuevas formas de comunicación e interacción con los usuarios (reales o virtuales) y de su constante capacidad de innovar y de su dominio de las TIC.

\section{Notas}

(1) El ranking, que reconoce a la Universidad Nacional Autónoma de México (UNAM), como la mejor de América Latina, se puede consultar en la Página http://www.webometrics.info.

Este ranking está avalado por una de las principales organizaciones de investigación básica de Europa, el CSIC, adscrito al Ministerio de Ciencia y Tecnología de España. La posición en el ranking resume el rendimiento global de la Universidad, aporta información para estudiantes o profesores, y refleja el compromiso académico con la diseminación del conocimiento científico. Posee una cobertura mayor que otros similares, ya que además de considerar los resultados de investigación, también usa referentes que muestran mejor la calidad global de las instituciones académicas y de investigación del mundo entero.

\section{Referencias}

Arroyo Vázquez, Natalia y José A Merlo Vega (2006). La biblioteca como usuaria de la web 2.0 http://eprints.rclis.org/9787/1/Arroyo\&Merlo_FESABID07 .pdf

Castells, Manuel. (1999). La era de la información: economías, sociedad y cultura. Vol. I La sociedad red. Madrid: Siglo Veintiuno Editores.

Castells, Manuel. (2003). La Galaxia Internet. Barcelona: Edición bolsillo $363 \mathrm{p}$.

Habib, M. C. (2006). Toward Academic Library 2.0: Development and Application of a Library 2.0 Methodology: Master's Paper for the Master of Science in Library Science degree. $49 \mathrm{p}$.

Islas Carmona, Octavio (2008). El prosumidor: el actor comunicativo de la sociedad de la ubicuidad. // Razón y Palabra. 61. http://www.razonypalabra.org.mx/espejo/ 2008/mar15.html

Kerchiove, Derrick de (2005). Los sesgos de la electricidad. Lección inaugural del curso académico 2005-2006 de la UOC. Barcelona: UOC, 2005. http://www.uoc.edu/inau gural05/esp/kerchove.pdf

Lee King, David (2007). Library 2.0 ripples: another go at the graph. August 24 http://www.davidleeking.com/2007/ .../library-20-ripples-another-go-at-the-graph

Margaix-Arnal, D. (2007). Conceptos de web 2.0 y biblioteca 2.0: origen, definiciones y retos para las bibliotecas actuales. // El profesional de la información. 16:2 (2007) 95-106.

Messner, Dirk. (1999). Del Estado céntrico a la «sociedad de redes: nuevas exigencias a la coordinación social. // Norbert Lechner, R. Milán y F. Valdés (coords.). Reforma del Estado y Coordinación Social. México IIS (UNAM). Plaza y Valdés.

OCDE (2007). Participative Web and User-created Content: Web 2.0, Wikis and social Networking Directorate for Science, Technology and Industry. OCDE: Paris.

Rheingold, Howard (2002). Reputation. Smart Mobs: the next social revolution. 113-132

Villouta Hurtado, Omar (2008). Conformación de redes digito-sociales: usos de la web 2.0. // Razón y palabra. 63 (2008). 
Zitt, Francisca (2007). Web 2.0 analogía de la selva y el desierto October 12, Traducción de Johnson, Steven (2007). "Llega la Web 2.0" // Discover 26 (10) October 2005. 